\title{
Statistical Analysis of Support Thickness and Particle Size Effects in HRTEM Imaging of Metal Nanoparticles
}

Stephen D. House ${ }^{\mathrm{a}^{*}}$, Cecile S. Bonifacio ${ }^{\mathrm{a}}$, Ross V. Grieshaber ${ }^{\mathrm{a}}$, Long Li ${ }^{\mathrm{a}, 1}$, Zhongfan Zhang ${ }^{\mathrm{a}}$, Jim Ciston ${ }^{\mathrm{b}}$, Eric A. Stach ${ }^{\mathrm{c}}$, and Judith C. Yang ${ }^{\mathrm{a}}$

${ }^{a}$ Chemical and Petroleum Engineering, and Physics, University of Pittsburgh, Pittsburgh, PA 15261, USA.

${ }^{\mathrm{b}}$ National Center of Electron Microscopy, Molecular Foundry, Lawrence Berkeley National Laboratory, Berkeley, CA 94720, USA.

${ }^{c}$ Center for Functional Nanomaterials, Brookhaven National Laboratory, Upton, NY 11973, USA.

${ }^{1}$ Present Address: RJ Lee Group, Inc., Monroeville, PA 15146, USA

\section{*Corresponding Author:}

Stephen D. House

Department of Chemical and Petroleum Engineering University of Pittsburgh

940 Benedum Hall

3700 O'Hara Street

Pittsburgh, PA, 15261 USA

$\mathrm{Ph}: 847-250-7357$

Email: sdh46@pitt.edu

Key words: HRTEM, support effect, nanoparticles, particle size, image artifacts, Cs aberration 


\begin{abstract}
High-resolution transmission electron microscopy (HRTEM) examination of nanoparticles requires their placement on some manner of support - either TEM grid membranes or part of the material itself, as in many heterogeneous catalyst systems - but a systematic quantification of the practical imaging limits of this approach has been lacking. Here we address this issue through a statistical evaluation of how nanoparticle size and substrate thickness affects the ability to resolve structural features of interest in HRTEM images of metallic nanoparticles on common support membranes. The visibility of lattice fringes from crystalline Au nanoparticles on amorphous carbon and silicon supports of varying thickness was investigated with both conventional and aberration-corrected TEM. Over the 1-4 nm nanoparticle size range examined, the probability of successfully resolving lattice fringes differed significantly as a function both of nanoparticle size and support thickness. Statistical analysis was used to formulate guidelines for the selection of supports and to quantify the impact a given support would have on HRTEM imaging of crystalline structure. For nanoparticles $\geq 1 \mathrm{~nm}$, aberration-correction was found to provide limited benefit for the purpose of visualizing lattice fringes; electron dose is more predictive of lattice fringe visibility than aberration correction. These results confirm that the ability to visualize lattice fringes is ultimately dependent on the signal-to-noise ratio of the HRTEM images, rather than the point-to-point resolving power of the microscope. This study provides a benchmark for HRTEM imaging of crystalline supported metal nanoparticles and is extensible to a wide variety of supports and nanostructures.
\end{abstract}

\title{
1. Introduction
}

Recent years have seen a proliferation of advanced nanoparticle synthesis techniques e.g., top-down, bottom-up, micellar methods, and electro-steric/static stabilization - enabling 
ever greater control over their size, morphology, and composition [1-4]. Nanoparticles can now be controllably produced that are only a few nanometers or below in size - ultra-small nanoparticles, with sub-nanometer aggregates of fewer than 20 atoms often called clusters. In this size range, the nanoparticles exhibit unusual size-dependent properties - e.g., electrical, magnetic, optical, and catalytic - and with it the potential for significant advancements in a vast array of fields, such as catalysis [5]. Taking advantage of these properties requires the accurate characterization of synthesized nanostructures at the relevant length scales (nanometer and subnanometer) to provide an understanding of their structure and behavior. This is important for the development of new and improved synthesis techniques for optimizing these structures for the desired performance, e.g., catalytic activity and selectivity [6]. Additionally, size-distribution statistics obtainable by characterization tools that provide local information are necessary for the interpretation of data from ensemble-averaged techniques (e.g., extended X-ray absorption fine structure (EXAFS), where coordination number is a crucial parameter $[7,8])$ ) that are often used in nanoparticle analysis.

Transmission electron microscopy (TEM) is a powerful and versatile tool for providing morphological, size (and distribution), and chemical information of individual nanoparticles with sub-unit-cell spatial resolutions, essential for determining structure-function relationships. Advancements in spherical aberration $\left(\mathrm{C}_{\mathrm{s}}\right)$ correcting hardware have delivered a significant increase in resolution $[9,10]$, rendering atomic-scale imaging of nanocrystal morphology a routine affair.

High-resolution TEM (HRTEM) imaging of nanometer-sized structures, however, can face a variety of challenges due to the small size, including beam damage, limited statistics, low signal-to-noise, and imaging artifacts arising from their finite nature $[5,11,12]$. In a previous 
work, we identified critical imaging issues - such as electron beam-induced changes, delocalization effects, inherent visibility of lattice fringes, and the role of the support - in determining crystallinity in Pt nanoparticles using TEM [13]. Despite the detrimental impact supports can have on image contrast [14], their presence is unavoidable, whether in the form of TEM grid membranes or as an intrinsic part of the material system itself. As an example of the latter, metal nanoparticles supported by high surface area materials are common heterogeneous catalysts and important to myriad industrial processes [15]. Model systems of supported metal nanoparticles on planar substrates is a common approach for exploring the microscopic processes otherwise obscured by the complexity of these materials [16]. McBride et al. [17] indicated that conventional TEM imaging of ultra-small NPs can be limited by particle diameters of greater than $3 \mathrm{~nm}$ due to the contrast from amorphous carbon support films. For such reasons, graphene membranes (being a single layer of carbon atoms) have garnered attention as the "ultimate" support for TEM imaging of nanoparticles [14, 17, 18]. Barring a major fabrication breakthrough, more conventional TEM films - e.g., amorphous carbon, silicon, or SiN - will likely remain ubiquitous due to cost, ease of use, durability, and versatility. In addition, nanoparticles generally do not adhere as well to graphene, leading to increased movement under the electron beam, which can hamper high-resolution measurements. Nonetheless, in spite of the awareness regarding this issue, the critical thicknesses at which the contrast from commonly used TEM supports start to interfere with TEM imaging of the sample of interest has not been systematically quantified. This will only become more important as sub-nanometer nanoparticle/cluster sizes of a few atoms enabled by the advancement in synthesis methods approach the limits of HRTEM characterization. In addition, understanding the effect of the support on TEM imaging of catalyst systems is necessary to accurately distinguish such imaging 
artifacts from actual structural evolution occurring in supported nanoparticles under environmental conditions. In situ TEM, which enables the observation of structural changes under environments - e.g., temperature, gaseous atmosphere, liquid - approaching those of working conditions [19-23], faces additional imaging challenges, as the presence of liquid, gas, and/or windows from closed-cell holders can greatly reduce signal intensity.

Here we present a statistical study of the effect of support thickness on HRTEM imaging of ultra-small nanoparticles on commonly used support membranes of varying thickness composition. The metric quantified in this analysis is the visibility of nanoparticle lattice fringes, as these features convey information about the crystalline structure of the particles. Since this outcome is categorical - the fringes can only be either visible or not-visible - rather than continuous, logistic regression models were applied to estimate the probabilities of successfully visualizing lattice fringes. It is important to note that this study does not directly address the interpretability of the experimental lattice contrast, only the binary metric of observability. A systematic examination of gold nanoparticles on various supports was performed to obtain sizedependent statistics of lattice fringe visibility as a function of support thickness. Au nanoparticles were selected as - based on previous work in Ref. $[13,24]$ and the sizes of nanoparticles measured in this study - it is reasonable to assume all the examined nanoparticles were crystalline and that lack of lattice fringe visibility was due entirely to imaging limitations rather than changes in nanoparticle morphology. Additionally, Au nanoparticles do not oxidize upon exposure to air, avoiding structural changes that would have complicated the analysis. In this work, quantification of the support effect on the ability to image nanoparticles was performed to verify the nanoparticle size dependence and determine critical size limits for a given support thickness. This investigation was carried out on both conventional and aberration-corrected 
TEM, to compare the respective limits of crystalline lattice fringe visualization. The method introduced here is broadly applicable to a wide variety of supports and crystalline nanostructures.

\section{Materials and Methods}

The TEM supports used for this study included $2 \mathrm{~nm}$ thick ultrathin carbon film TEM grids (Ted Pella Inc.) and 5 and $9 \mathrm{~nm}$ thick, amorphous-silicon window TEM grids (Structure Probe Inc. / SiMPore Inc.). The nominal thickness of the grids given by the manufacturers $(2,5$, and $9 \mathrm{~nm}$ ) were independently measured via EELS using a JEOL JEM 2100F TEM/STEM equipped with a Gatan GIF Tridiem. The log-ratio method was employed, as detailed by Egerton [25] (see also Supplemental Materials S1). Twenty measurements were made across each film, with the thicknesses averaging $6.8 \pm 1.8,8.7 \pm 1.3$, and $12.1 \pm 0.3 \mathrm{~nm}$, respectively. It is likely the carbon film in particular is overestimated due to build-up from the stationary focused beam; longer acquisition times yielded correspondingly higher thickness values. The silicon films did not exhibit this same sensitivity to acquisition duration. Regardless of potential systematic overestimation in the absolute thickness, the relative thicknesses of the films match fairly well with the nominal values.

Au nanoparticles (NPs) were thermally evaporated onto these TEM support grids at $10^{-6}$ torr with the substrate held at room temperature. Supplemental Materials S2 is a representative HRTEM image showing the size range of the resulting Au NPs. Based on the range of observed NP sizes and previous studies of Au NP [13, 24], it was assumed that all the examined NP were crystalline.

TEM characterization was carried-out using a Philips CM300 FEG TEM/STEM $(\mathrm{Cs}=$ $0.65 \mathrm{~mm})$ and the double-aberration-corrected FEI TEAM 0.5 TEM/STEM $(\mathrm{Cs}=0.005 \mathrm{~mm})$, both with UltraTwin pole pieces and operated at $300 \mathrm{kV}$ without monochromation to ensure 
comparable information limits $(0.8 \AA)$. The TEAM 0.5 microscope is a modified FEI Titan TEM/STEM equipped with two CEOS hexapole-type spherical aberration correctors $(0.8 \AA$ information limit without monochromation [26]). Appropriate illumination conditions were established wherein a constant/low electron dose did not modify the Au NP during TEM examination.

Through-focal image series - i.e., focal-series HRTEM (FS-HRTEM) - of the nanoparticles were acquired to determine the presence of lattice fringes. The focus step sizes (the amount the defocus was varied between images in a series) were $-3.0 \mathrm{~nm}$ for the TEAM 0.5 microscope and $-4.1 \mathrm{~nm}$ for the CM300. An example FS-HRTEM series is included in Supplemental Materials S3. The average electron doses, calculated from the micrographs, for the focal series taken on the TEAM 0.5 were $6.7 \times 10^{4}, 9.9 \times 10^{4}$, and $7.6 \times 10^{4} \mathrm{e}^{-} / \mathrm{nm}^{2}$ for the 2,5 , and $9 \mathrm{~nm}$ films. The average dose for the CM300 focal series was $5.9 \times 10^{5} \mathrm{e}^{-/ \mathrm{nm}^{2}}$. For all series, the exposure time for each image was 1 second.

These focal series were not reconstructed for the purpose of investigating the complex exit wave, but were instead analyzed serially to ensure that all structurally relevant spatial frequencies were adequately sampled to avoid zero crossings of the phase contrast transfer function. The crystallinity of the Au NPs was determined in a similar manner as the order versus disordered criteria of lattice fringe observations described in our previous work [13]. Diffractogram analysis was used to verify that observed lattice fringes corresponded to real structural frequencies. The visibility of the lattice fringes in the NPs during TEM imaging was addressed by considering the overlap between the lattice spacing and the information limit of the instrument. In this case, the instrument's information limit must resolve the main interplanar spacings of a material obtained along all the major, low-index zone axis orientations. The 
complex interrelationships between the HRTEM images and details of the NP orientation discussed in previous studies [27-32] were considered here.

The NP sizes (diameters) were measured from the acquired FS-HRTEM images and binned by size (rounded up). The visibility or obfuscation of lattices fringes in a particle was treated as a binary response independent of spatial frequency ( 1 for visible, 0 for obfuscated) to yield a binomial distribution for each support type. Upon these distributions, linear regression analysis or logistic regression analysis, as applicable, was performed in the statistical computing environment $R$ (version 3.2.2) [33] to estimate the probability that crystalline features could be successfully imaged for a given nanoparticle size and support thickness. In datasets where no statistically significant change in lattice fringe visibility was observed over the range of NP sizes present, a linear regression model was applied. In all other cases, a logistic regression model was used. A logistic regression was selected for this analysis because the response variable (lattice fringe visibility) is a categorical (either visible or obfuscated), rather than continuous, variable, and as such violates the assumption of linearity in a normal regression. What is being estimated is the probability of the event (lattice fringe visibility) occurring. The logistic regression also evaluates the relative importance or impact of the independent variable(s) in predicting the response and whether there are any interactions between the predictors.

For the analysis in this work, each support type was fitted independently, using NP size as the sole predictor variable. The linear regression model was based on the following equation:

$$
F=\beta_{0}+\beta_{1} x
$$

where $F$ is the fraction of the observed NPs with visible lattice fringes, $\beta_{0}$ is the intercept, $x$ is the predictor variable (NP size), and $\beta_{1}$ is the coefficient for the predictor variable. The logistic regression model (Equation 2), using the logit transformation (Equation 3), is given by: 


$$
\begin{gathered}
P=\frac{e^{\beta_{0}+\beta_{1} x}}{1+e^{\beta_{0}+\beta_{1} x}} \\
\operatorname{logit}(P)=\ln \left(\frac{P}{1-P}\right)=\beta_{0}+\beta_{1} x
\end{gathered}
$$

where $P$ is the estimated probability of the event to occur (i.e., visualization of lattice fringes in the NP), and the other terms are the same as in the linear regression (Equation 1). The generalized forms of these regression models, allowing for multiple predictor variables, are discussed in Supplemental Material S4. The linear regression models were evaluated using the generated residual standard error and by visual inspection. The logistic regression models were evaluated by the resulting $\mathrm{p}$-values and calculated pseudo $\mathrm{R}^{2}$ (1- ratio of residual deviance and null deviance). The p-value measures the significance of the variable by estimating the probability of obtaining a coefficient with a magnitude as large as we observed if the variable has no effect on the outcome (null hypothesis) [34]. A p-value of less than 0.05 is considered statistically significant.

\section{Results}

Multiple HTREM focal series were acquired on each support type. From these series, representative images of which are shown in Figure 1 for the (a) $2 \mathrm{~nm}$, (c) $5 \mathrm{~nm}$, and (e) $9 \mathrm{~nm}$ thick supports, respectively, imaged using the aberration-corrected TEM, The sizes of nanoparticles were measured and the presence or absence of distinguishable lattice fringes were determined. The observed Au NPs ranged in size from $\sim 1.1$ to $4 \mathrm{~nm}$ for the 2 and $5 \mathrm{~nm}$ supports, and up to $8 \mathrm{~nm}$ for the $9 \mathrm{~nm}$-thick support. Higher magnification examples of individual NP exhibiting visible lattice fringes, indicated by arrows in Figure 1, are inset. The NPs were randomly oriented with respect to the electron beam. The corresponding histograms of 
nanoparticle size, categorized by lattice fringe visibility, are provided in Figure 1b,d,f. The total number of NPs measured, N, is listed in the upper right or each histogram.

The experimental NP data (symbols) - the fraction of NP with visible lattice fringes and their corresponding fits (lines) are plotted for comparison in Figure 2 as a function of nanoparticle size. The shaded regions are the $95 \%$ confidence intervals of the regression models. Each fitted curve provides an estimate of the probability that the lattice fringes of a nanoparticle of a given size will be visible in an HRTEM image. For the $2 \mathrm{~nm}$ amorphous carbon support, only a single mid-sized ( $2 \mathrm{~nm}$ diameter) nanoparticle out of $132 \mathrm{NPs}$ was observed with obfuscated lattice fringes. As such, the data were fit with a linear regression. The $5 \mathrm{~nm}$ and $9 \mathrm{~nm}$ amorphous silicon supports exhibited a broader range of obfuscated particles and were each fit with a logistic regression. The resulting fitting coefficients and fit metrics are summarized in Table 1. Summaries of the model fits are included in Supplemental Materials S4.

Table 1. Fit parameters and metrics for the linear regression $(2 \mathrm{~nm}$ a-carbon and $5 \mathrm{~nm}$ a-silicon uncorrected) and logistic regression (5 and $9 \mathrm{~nm}$ a-silicon), plotted in Figures 2 and 4.

\begin{tabular}{ccccc}
\hline $\begin{array}{c}\text { Linear } \\
\text { regression }\end{array}$ & $\mathbf{B}_{\mathbf{0}}$, Intercept & $\mathbf{B}_{\mathbf{1}}$, Coefficient & \multicolumn{2}{c}{ Residual Standard Error } \\
\hline $2 \mathrm{~nm}$ a-carbon & 0.995 & $6.92 \times 10^{-4}$ & 0.0103 & \\
\hline $\begin{array}{c}5 \mathrm{~nm} \text { a-silicon } \\
\text { uncorrected }\end{array}$ & 0.979 & $6.01 \times 10-3$ & 0.0233 & \\
\hline $\begin{array}{c}\text { Logistic } \\
\text { regression }\end{array}$ & $\mathbf{B}_{\mathbf{0}}$, Intercept & $\mathbf{B}_{\mathbf{1}}$, Coefficient & p-value & Pseudo-R $^{\mathbf{2}}$ \\
\hline $5 \mathrm{~nm}$ a-silicon & -1.24 & 1.93 & $4.27 \times 10^{-4}$ & 0.673 \\
$9 \mathrm{~nm}$ a-silicon & -3.62 & 3.04 & $1.07 \times 10^{-7}$ & 0.955 \\
\hline
\end{tabular}

The linear regression fit to the $2 \mathrm{~nm}$ a-carbon film data shows good agreement by visual inspection and the low $(<0.01)$ standard error. The fit is not statistically significantly different from a horizontal line through the mean of the experimental values, indicating that over the size range of nanoparticles observed $(1-4 \mathrm{~nm})$, the visibility of lattice fringes is not impacted by the 
support. The extremely low p-values $(<0.0005)$ of the logistic regressions of the 5 and $9 \mathrm{~nm}$ thick films, however, indicates a significant dependence of lattice fringe visibility on nanoparticle size for the given support type and thickness. This means that the supports strongly affect the ability to image these particles (i.e. the "support-effect") and the degree of this effect is a function of particle size. The high pseudo- $\mathrm{R}^{2}$ value of the $9 \mathrm{~nm}$ fit signifies a good fit of the logistic regression model and that almost all the variation in lattice fringe visibility in the dataset can be accounted for by the support-effect. The pseudo- $\mathrm{R}^{2}$ of the $5 \mathrm{~nm}$ fit is lower but still clearly shows that the support-effect is the dominant factor. The greater variance as the particle size decreases (Figure 2) is at least in part an artifact of the low counting statistics due to having fewer smaller nanoparticles for measurement. This variance is taken into account by the $95 \%$ confidence intervals, seen in Figure 2, in which a clear difference in response (lattice fringe visibility) between the different substrate thicknesses as nanoparticle size decreases.

The NPs on the $5 \mathrm{~nm}$-thick a-silicon support were also examined using a conventional, non-aberration-corrected TEM. An example micrograph and the size distribution histogram from the dataset are shown in Figure 3. Though not as sharp in fine detail, the NPs in the images from the uncorrected micrographs exhibited a higher contrast against the background membrane than in the aberration-corrected images. Like with the $2 \mathrm{~nm}$ a-carbon support, only a single NP (this time $1.5 \mathrm{~nm}$ in diameter) out of 158 with obfuscated lattice fringes was observed(c.f. Figure $3 \mathrm{~b}$ ), so a linear regression fit was the most logical choice. The fit is summarized in Table 1 and the uncorrected and the corresponding aberration-corrected data and fits are plotted in Figure 4. Again, the low standard error and goodness of fit by visual inspection of the linear model to the uncorrected data indicate that the lattice fringe visibility over the 1-4 nm size range was not impacted to any statistically significant degree by the presence of the support. This increase in 
probability of visualizing lattice fringes using the uncorrected TEM was observed with the other supports, too, though their sample sizes were too low for any meaningful statistical.

\section{Discussion}

\subsection{Impact of Support Thickness}

Although lattices fringes could be visualized at all nanoparticle sizes for all the supports examined, the probably of their visibility as nanoparticle size decreased diminished significantly with increasing support thickness. In particular, the 5 and $9 \mathrm{~nm}$ thick supports diverged from the $2 \mathrm{~nm}$ thick support beginning with NPs about $2.5 \mathrm{~nm}$ in size, while the 5 and $9 \mathrm{~nm}$ thick supports began to diverge from each other below a NP size of $\sim 2 \mathrm{~nm}$ (c.f. Figure 2). The critical nanoparticle sizes (determined from the fits) for a given probability of lattice fringe visibility for each support type are summarized in Table 2.

Table 2. Critical nanoparticle sizes for three different probabilities of lattice fringe visibility, as calculated from the fits (Table 1 and Figure 2).

\begin{tabular}{ccccc}
\hline & \multicolumn{4}{c}{ Critical Nanoparticle Size (nm) } \\
Support & $\mathbf{5 0 \%}$ Visibility & $\mathbf{7 5 \%}$ & $\mathbf{9 0 \%}$ & $\mathbf{9 5 \%}$ \\
\hline $\mathbf{2} \mathbf{~ n m}$ a-carbon & $\mathrm{n} / \mathrm{a}^{*}$ & $\mathrm{n} / \mathrm{a}^{*}$ & $\mathrm{n} / \mathrm{a}^{*}$ & $\mathrm{n} / \mathrm{a}^{*}$ \\
$\mathbf{5} \mathbf{~ n m}$ a-silicon & $\mathrm{n} / \mathrm{a}^{\dagger}$ & 1.2 & 1.8 & 2.2 \\
$\mathbf{9} \mathbf{~ n m}$ a-silicon & 1.2 & 1.6 & 1.9 & 2.2 \\
\hline
\end{tabular}

*The visibility of lattice fringes in NPs over the range of NP sizes observed (1-4 nm) was not impacted by the presence of the support film, retaining full visibility. ${ }^{\dagger}$ This value lies outside the size range of NPs observed in this study, and so is not necessarily accurate.

The ultrathin $2 \mathrm{~nm}$ amorphous carbon support is clearly the ideal choice, if possible, as no degradation of lattice fringe visualization was observed over the NP size range examined. However, this is not always a viable option for all TEM experiments, as such films possess lower durability (e.g., to $\mathrm{O}_{2}$ plasma, heating, mechanical stress, etc). For nanoparticles $2 \mathrm{~nm}$ or larger in size, support films up to $9 \mathrm{~nm}$ thick will perform reasonably similar, with $>90 \%$ probability to visualize lattice fringes. Below this size, a statistically significant degradation arises. The $9 \mathrm{~nm}$ 
thick amorphous silicon support fell to $75 \%$ visibility once the NP size reaches $1.6 \mathrm{~nm}$, while the $5 \mathrm{~nm}$ support maintained this level down to $1.2 \mathrm{~nm}$ nanoparticles. NP visibility drops off rapidly below this point, as the distribution is sigmoidal, with an inflection point at $50 \%$ visibility probability. This $50 \%$ visibility occurs at $1.2 \mathrm{~nm}$ for the $9 \mathrm{~nm}$ thick support, and somewhere below $1 \mathrm{~nm}$ for the $5 \mathrm{~nm}$ thick support.

It is important to note that the listed values are intended as guidelines, rather than exact dictates. The low NP counts for the smaller NP sizes introduce a greater uncertainty in the fit in those regions, as indicated by the $95 \%$ confidence intervals. Additionally, natural variation between different microscopes and/or sample films will alter the results slightly. That said, these critical sizes still provide a good estimate of how much one could expect a given support to impact their NP imaging. This statistical analysis provides a helpful guidance when considering which support to use given the experimental parameters and desired NP size(s). It also offers a statistically quantified estimation of the "support effect" artifacts that would be introduced in, for example, studies of crystallinity in smaller nanoparticles - i.e., the likelihood the observed disorder in a NP is real vs. merely being obfuscated by the support. The results of this study indicate that the successful high-resolution imaging of such features is ultimately limited in practice by the signal-to-noise ratio (SNR) induced by the support rather than by the point resolution or information limit of the microscope. This obfuscation of imaging NPs is of extra concern for in situ HRTEM measurements, as the SNR is further reduced by the presence of liquids, gases, and/or the windows of close-cell holders. This work provides the first statistical quantification of the support-effect and a framework for gauging it that can be easily applied to other systems, including support types as well as gas/liquid environments and windows. 


\subsection{Limited Impact of Aberration Correction}

Compared to the aberration-corrected images, the nanoparticles imaged using the uncorrected TEM (c.f. Figure 1c and Figure 3a) exhibited a higher contrast against the background rendering them easier to distinguish. Further, as evidenced by Figure 4, the HRTEM imaging using the aberration-corrected versus uncorrected TEM differed notably in their ability to resolve the lattice fringes of nanoparticles on a given support. On the $5 \mathrm{~nm}$ thick support, the difference grew significantly for imaging NPs $\sim 2 \mathrm{~nm}$ and smaller. This behavior may primarily be attributed to the increased (approximately six times higher) electron dose of the uncorrected focal series images, as in this dosage range the signal-to-noise ratio of simulated HRTEM images was found to be nearly linear with dose [35]. While SNR does not always directly correspond to visibility, all else equal a higher SNR should improve the visibility. In addition to confirming this expectation, comparison between the $5 \mathrm{~nm}$ experiments also indicates that aberration correction does not appreciably improve the ability to visualize lattice fringes in such nanoparticles; the SNR is more important.

Given how significantly the uncorrected TEM outperformed the aberration-corrected microscope in this test with an electron dosage less than an order of magnitude higher, is possible that there are additional secondary attributes of uncorrected TEM that contribute to the improved lattice fringe visibility. This behavior may be explained by the contrast transfer function (CTF), computed using MacTempasX [36], for the TEAM 0.5 (Figure $5 \mathrm{a}-\mathrm{b}$ ) and CM300 (Figure 5c-d) microscopes near the Scherzer defocus condition. Although the two microscopes share the same incoherent envelope, it is sampled differently by the coherent function of each. The contrast is higher at low spatial frequencies in the uncorrected CTF, and the tail does not fall off as quickly at higher spatial frequencies; these aspects persist at other 
defoci. The recurrent oscillations of the uncorrected CTF are likely providing a fuller sampling of the incoherent envelope, yielding a higher probability of a particular fringe spacing passing through a CTF maximum, and thus transferred strongly to the imaging plane. Consequently, the lattice fringes appear in images as oscillations on top of a dark background where the speckle contrast of the amorphous background is suppressed, yielding a higher signal to noise ratio. In the aberration-corrected case, the nanoparticle has a similar overall contrast as the background; the lattice oscillations in contrast are directly in competition with the amorphous speckle, leading to a lower relative contrast. Furthermore, uncorrected TEM imaging is subject to greater delocalization, extending the fringes outside the formal nanoparticle boundary, which may make their presence easier to notice by eye. This delocalization also means the NP sizes are likely to be overestimated for the uncorrected case, implying that the uncorrected TEM is possibly even more advantageous for lattice fringe visibility than indicated here. It should be noted that in an aberration-corrected TEM the value of Cs can be adjusted using the corrector, shifting the position of the CTF crossover(s) and enabling a CTF similar to an uncorrected TEM to be obtained, if so desired.

The improvement in lattice fringe visibility, however, comes at a non-trivial cost. The greater number of zero crossings in the uncorrected CTF hinders the interpretability of the images. Aberration correction also decreases delocalization errors and produces a finer point resolution. Thus, if the visibility of lattice fringes (or similar) is the primary desired feature, or if other aspects of the experiment preclude the use of thinner supports, it may actually be beneficial to employ an uncorrected TEM or intentionally increase the value of Cs in an aberrationcorrected TEM. These results are only directly applicable to experiments attempting to distinguish ordered from disordered NPs, and do not directly extend to the identification of 
different crystal structures. In order to address the question of structure determination, further analysis of the relationship between spatial frequency and observability on various supports are required. This will be the subject of future work.

\section{Conclusions}

In summary, we performed a systematic study of the impact of commonly used TEM support films - amorphous carbon and silicon - on the ability to resolve the internal structure, in the form of lattice fringes, of ultra-small nanoparticles. The results of this work establish a quantitative statistical benchmark for HRTEM imaging of such aspects in nanoparticles on supports. Over the range of nanoparticles observed - as small as $1 \mathrm{~nm}$ - the ultrathin $2 \mathrm{~nm}$ carbon support had no negative impact on the visualization of lattice fringes. The performance of thicker 5 and $9 \mathrm{~nm}$ amorphous silicon, however, was found to fit well with logistic regression models. For NP around $2 \mathrm{~nm}$ and larger, the thicker 5 and $9 \mathrm{~nm}$ supports retained the ability to visualize lattice fringes with $>90 \%$ probability. Below this, however, the three supports diverge significantly. The probability of visualization drops to $75 \%$ in nanoparticles $\leq 1.2 \mathrm{~nm}$ and $\leq 1.6$ $\mathrm{nm}$ in size for the 5 and $9 \mathrm{~nm}$ a-silicon supports, respectively, with $1.2 \mathrm{~nm}$ NPs falling to $50 \%$ on the thicker $9 \mathrm{~nm}$ grid.

Uncorrected TEM with a moderately higher electron dose was found to improve lattice fringe visibility significantly - e.g., full visualization over the size range (1-4 nm) examined on the $5 \mathrm{~nm}$ substrate - compared to the equivalent aberration-corrected counterpart. This gain occurs at the cost of the other benefits of aberration correction, such as higher spatial resolution and improved interpretability, but may prove a preferable trade-off for some experiments and 
materials systems. It also confirms that, in practice, the ultimate limit for resolving lattice fringes is the signal-to-noise ratio rather the point resolution or information of the microscope.

This work provides quantitative guidelines for the selection of support, to be considered along with other experimental requirements, to ensure the greatest chances of obtaining the information desired during TEM examination of NPs as a function of NP size. The results are also of particular value for avoiding ambiguity arising from imaging artifacts that could otherwise obscure real changes in nanoparticle structure, by offering a statistical estimation of the support's impact on such features. While the NP size imaging to support thickness guidelines reported are generalizable to other high $\mathrm{Z}$ metal nanostructures dispersed on low $\mathrm{Z}$ supports in this thickness range, the benchmarking methodology presented in this paper can be easily extended to other supports and materials.

\section{Acknowledgements}

This research project was supported by DOE BES under Contract No. DE-FG02- 03 ER15476. The electron microscopy was performed at the Molecular Foundry at Lawrence Berkeley National Lab which is supported by the Office of Basic Energy Sciences of the US Department of Energy under Contract No. DE-AC02-05CH11231. The authors kindly acknowledge Dr. Abhay Gautam for technical assistance with the evaporation of Au nanoparticles onto various support grids. The authors would also like to acknowledge Allison Gardner (University of Illinois) and Jie Li (University of Pittsburgh) for their valuable suggestions and discussions on statistical analysis. E.A.S. acknowledges funding to the Center for Functional Nanomaterials, Brookhaven National Laboratory, which is supported by the U.S. Department of Energy, Office of Basic Energy Sciences, under Contract No. DE-AC02-98CH10886. 


\section{References}

[1] V. Amendola, M. Meneghetti, G. Granozzi, S. Agnoli, S. Polizzi, P. Riello, A. Boscaini, C. Anselmi, G. Fracasso, M. Colombatti, C. Innocenti, D. Gatteschi, C. Sangregorio, Top-down synthesis of multifunctional iron oxide nanoparticles for macrophage labelling and manipulation, Journal of Materials Chemistry, 21 (2011) 3803-3813.

[2] Y. Wang, Y. Xia, Bottom-Up and Top-Down Approaches to the Synthesis of Monodispersed Spherical Colloids of Low Melting-Point Metals, Nano Letters, 4 (2004) 2047-2050.

[3] B. Roldan Cuenya, J.R. Croy, S. Mostafa, F. Behafarid, L. Li, Z. Zhang, J.C. Yang, Q. Wang, A.I. Frenkel, Solving the Structure of Size-Selected Pt Nanocatalysts Synthesized by Inverse Micelle Encapsulation, Journal of the American Chemical Society, 132 (2010) 8747-8756.

[4] J. Patel, L. Nemcova, P. Maguire, W.G. Graham, D. Mariotti, Synthesis of surfactant-free electrostatically stabilized gold nanoparticles by plasma-induced liquid chemistry, Nanotechnology, 24 (2013) 245604.

[5] B.H. Kim, M.J. Hackett, J. Park, T. Hyeon, Synthesis, Characterization, and Application of Ultrasmall Nanoparticles, Chemistry of Materials, 26 (2013) 59-71.

[6] A.T. Bell, The Impact of Nanoscience on Heterogeneous Catalysis, Science, 299 (2003) 1688-1691.

[7] A.I. Frenkel, Applications of extended X-ray absorption fine-structure spectroscopy to studies of bimetallic nanoparticle catalysts, Chem. Soc. Rev., 41 (2012) 8163-8178.

[8] J.M. Thomas, J.-C. Hernandez-Garrido, Probing Solid Catalysts under Operating Conditions: Electrons or X-rays?, Angew. Chem. Int. Ed., 48 (2009) 3904-3907.

[9] M. Haider, S. Uhlemann, E. Schwan, H. Rose, B. Kabius, K. Urban, Electron microscopy image enhanced, Nature, 392 (1998) 768-769.

[10] H.H. Rose, Historical aspects of aberration correction, J. Electron Microsc., 58 (2009) 7785.

[11] Y. Li, D. Zakharov, S. Zhao, R. Tappero, U. Jung, A. Elsen, P. Baumann, R.G. Nuzzo, E.A. Stach, A.I. Frenkel, Complex structural dynamics of nanocatalysts revealed in Operando conditions by correlated imaging and spectroscopy probes, Nat Commun, 6 (2015).

[12] D.C. Bell, Y. Wu, C.J. Barrelet, S. Gradečak, J. Xiang, B.P. Timko, C.M. Lieber, Imaging and analysis of nanowires, Microscopy Research and Technique, 64 (2004) 373-389.

[13] L. Li, L.-L. Wang, D.D. Johnson, Z. Zhang, S.I. Sanchez, J.H. Kang, R.G. Nuzzo, Q. Wang, A.I. Frenkel, J. Li, J. Ciston, E.A. Stach, J.C. Yang, Noncrystalline-to-Crystalline Transformations in Pt Nanoparticles, J. Am. Chem. Soc., 135 (2013) 13062-13072.

[14] J.C. Meyer, C.O. Girit, M.F. Crommie, A. Zettl, Imaging and dynamics of light atoms and molecules on graphene, Nature, 454 (2008) 319-322.

[15] B.C. Gates, Supported Metal Clusters: Synthesis, Structure, and Catalysis, Chemical Reviews, 95 (1995) 511-522.

[16] J. Libuda, T. Schalow, B. Brandt, M. Laurin, S. Schauermann, Model studies in heterogeneous catalysis at the microscopic level: from the structure and composition of surfaces to reaction kinetics, Microchim Acta, 156 (2006) 9-20.

[17] J.R. McBride, A.R. Lupini, M.A. Schreuder, N.J. Smith, S.J. Pennycook, S.J. Rosenthal, Few-Layer Graphene as a Support Film for Transmission Electron Microscopy Imaging of Nanoparticles, ACS Appl. Mater. Interfaces, 1 (2009) 2886-2892.

S. House et al. 
[18] Z. Lee, K.-J. Jeon, A. Dato, R. Erni, T.J. Richardson, M. Frenklach, V. Radmilovic, Direct Imaging of Soft-Hard Interfaces Enabled by Graphene, Nano Letters, 9 (2009) 3365-3369.

[19] M. Delalande, M.J.F. Guinel, L.F. Allard, A. Delattre, R. Le Bris, Y. Samson, P. BayleGuillemaud, P. Reiss, L 10 Ordering of Ultrasmall FePt Nanoparticles Revealed by TEM In Situ Annealing, J. Phys. Chem. C, 116 (2012) 6866-6872.

[20] J. Matos, L.K. Ono, F. Behafarid, J.R. Croy, S. Mostafa, A.T. DeLaRiva, A.K. Datye, A.I. Frenkel, B. Roldan Cuenya, In situ coarsening study of inverse micelle-prepared Pt nanoparticles supported on $\gamma$-A12O3: pretreatment and environmental effects, PCCP, 14 (2012) 11457-11457. [21] Y.K. Mishra, V.S.K. Chakravadhanula, V. Hrkac, S. Jebril, D.C. Agarwal, S. Mohapatra, D.K. Avasthi, L. Kienle, R. Adelung, Crystal growth behaviour in Au-ZnO nanocomposite under different annealing environments and photoswitchability, J. Appl. Phys., 112 (2012) 064308064308.

[22] J. Park, H. Zheng, W.C. Lee, P.L. Geissler, E. Rabani, A.P. Alivisatos, Direct Observation of Nanoparticle Superlattice Formation by Using Liquid Cell Transmission Electron Microscopy, Acs Nano, 6 (2012) 2078-2085.

[23] J.R. Jinschek, Advances in the environmental transmission electron microscope (ETEM) for nanoscale in situ studies of gas-solid interactions, Chem. Commun., 50 (2014) 2696-2706. [24] L.D. Menard, H. Xu, S.-P. Gao, R.D. Twesten, A.S. Harper, Y. Song, G. Wang, A.D. Douglas, J.C. Yang, A.I. Frenkel, R.W. Murray, R.G. Nuzzo, Metal Core Bonding Motifs of Monodisperse Icosahedral Au13 and Larger Au Monolayer-Protected Clusters As Revealed by X-ray Absorption Spectroscopy and Transmission Electron Microscopy, Journal of Physical Chemistry B, 110 (2006) 14564-14573.

[25] R.F. Egerton, Electron Energy-Loss Spectroscopy in the Electron Microscope, 3rd ed., Springer US, New York City, 2011.

[26] U. Dahmen, R. Erni, V. Radmilovic, C. Ksielowski, M.-D. Rossell, P. Denes, Background, status and future of the Transmission Electron Aberration-corrected Microscope project, Philosophical Transactions of the Royal Society of London A: Mathematical, Physical and Engineering Sciences, 367 (2009) 3795-3808.

[27] J.A. Ascencio, C. Gutiérrez-Wing, M.E. Espinosa, M. Marín, S. Tehuacanero, C. Zorrilla, M. José-Yacamán, Structure determination of small particles by HREM imaging: theory and experiment, Surface Science, 396 (1998) 349-368.

[28] P.A. Crozier, S.C.Y. Tsen, J. Liu, C.L. Cartes, J.A. Perez-Omil, Factors affecting the accuracy of lattice spacing determination by HREM in nanometre-sized Pt particles, J. Electron Microsc., 48 (1999) 1015-1024.

[29] A.B. Flores, L.A. Robles, M.O. Arias, J.A. Ascencio, Small metal nanoparticle recognition using digital image analysis and high resolution electron microscopy, Micron, 34 (2003) 109118.

[30] P. Fraundorf, W. Qin, P. Moeck, E. Mandell, Making sense of nanocrystal lattice fringes, J. Appl. Phys., 98 (2005) 114308/114301-114308/.

[31] K. Koga, K. Sugawara, Population statistics of gold nanoparticle morphologies: direct determination by HREM observations, Surface Science, 529 (2003) 23-35.

[32] J. Zhang, Y. Tang, K. Lee, M. Ouyang, Nonepitaxial Growth of Hybrid Core-Shell Nanostructures with Large Lattice Mismatches, Science, 327 (2010) 1634-1638.

[33] R Core Team, R: A language and environment for statistical computing., in, R Foundation for Statistical Computing, Vienna, Austria, 2015.

[34] N. Zumel, J. Mount, Practical Data Science with R, Manning Publications Co., 2014.

S. House et al. 
[35] Z. Lee, H. Rose, O. Lehtinen, J. Biskupek, U. Kaiser, Electron dose dependence of signalto-noise ratio, atom contrast and resolution in transmission electron microscope images, Ultramicroscopy, 145 (2014) 3-12.

[36] R. Kilaas, MacTempasX version 2.4.25, in, Total Resolution LLC, 2015.

Figure 1. Example HRTEM (a,c,e) images from focal series of Au NPs deposited on (a) $2 \mathrm{~nm}$ thick a-carbon, (c) $5 \mathrm{~nm}$ a-silicon, and (e) $9 \mathrm{~nm}$ a-silicon films, acquired using the aberrationcorrected TEAM 0.5 microscope. Each inset shows a higher resolution image of a selected nanoparticle, indicated by an arrow, that exhibits visible lattice fringes. The corresponding size histograms for each support type are included in (b,d,f), respectively, divided up by NPs with visible (red) and obfuscated (white/cross-hatched) lattice fringes. The total number of NPs measured from each support type $(\mathrm{N})$ are listed in the upper right of the histogram.

Figure 2. Experimental measurements (symbols) of the fraction of observed nanoparticles with visible lattice fringes plotted along with their corresponding fits (lines) - linear for $2 \mathrm{~nm}$ acarbon and logistic for 5 and $9 \mathrm{~nm}$ a-silicon - as a function of nanoparticle size. The shaded regions are the $95 \%$ confidence intervals.

Figure 3. Example (a) HRTEM image from a focal series of Au NP on $5 \mathrm{~nm}$ thick a-silicon support, acquired using the uncorrected CM300 microscope. A higher resolution image of a selected nanoparticle, indicated by an arrow, with visible lattice fringes is shown in the inset. The corresponding size histogram of NPs with visible (red) and obfuscated (white/cross-hatched) is included in (b). The total number of NPs measured (N) was 158.

Figure 4. Comparison of experimental (symbols) and fitted (lines) lattice fringe visibility for the uncorrected (linear fit) and aberration-corrected (logistic fit) examinations of NPs on $5 \mathrm{~nm}$ thick a-silicon as a function of NP size. The shaded regions are the $95 \%$ confidence intervals.

Figure 5. Computed contrast transfer functions for the (a-b) aberration-corrected TEAM 0.5 and (c-d) uncorrected Philips CM300 TEMs near the Scherzer defocus condition. (b,d) are profiles of the 2D CTFs shown in $(\mathrm{a}, \mathrm{c})$, respectively. 

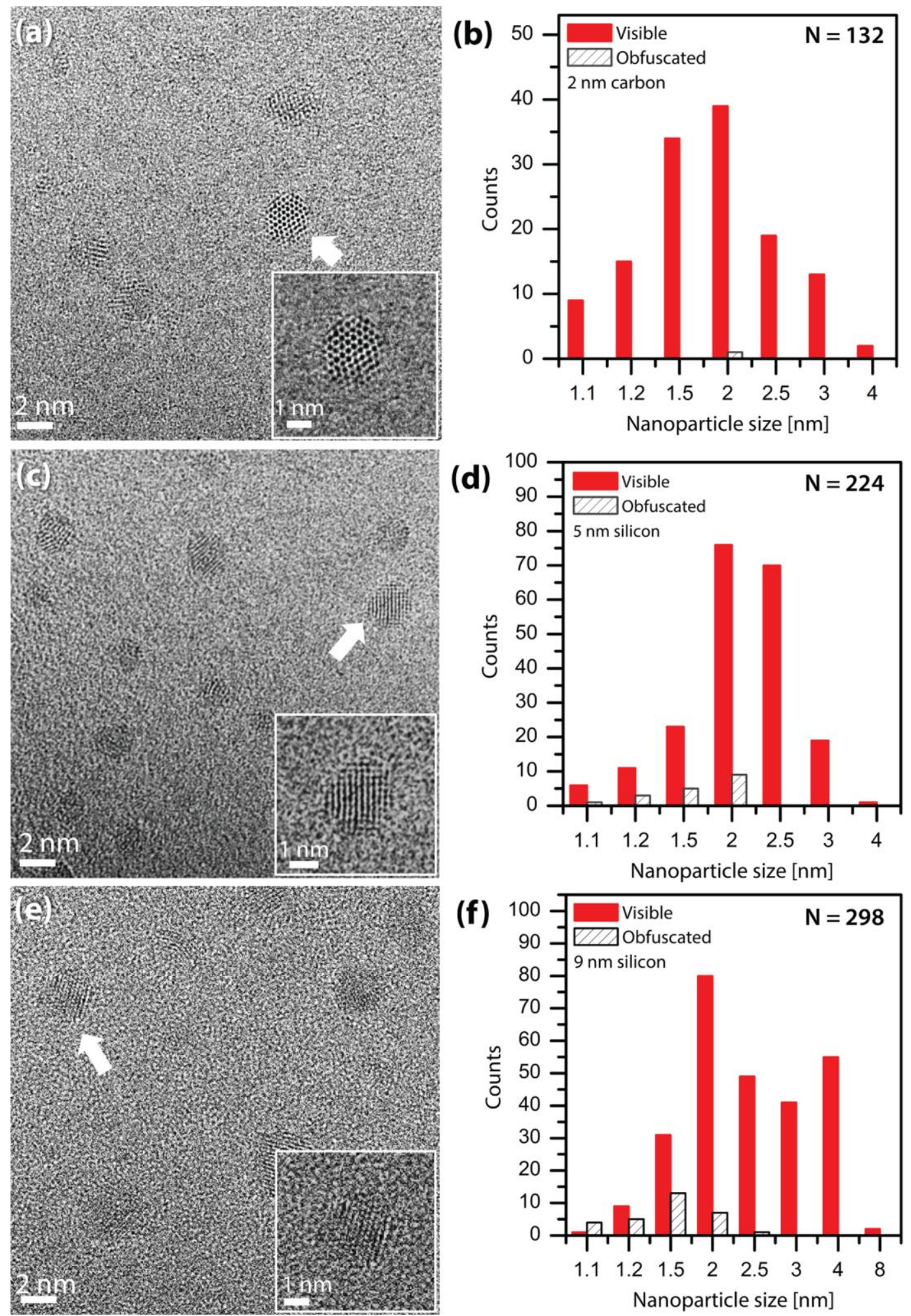

S. House et al. 


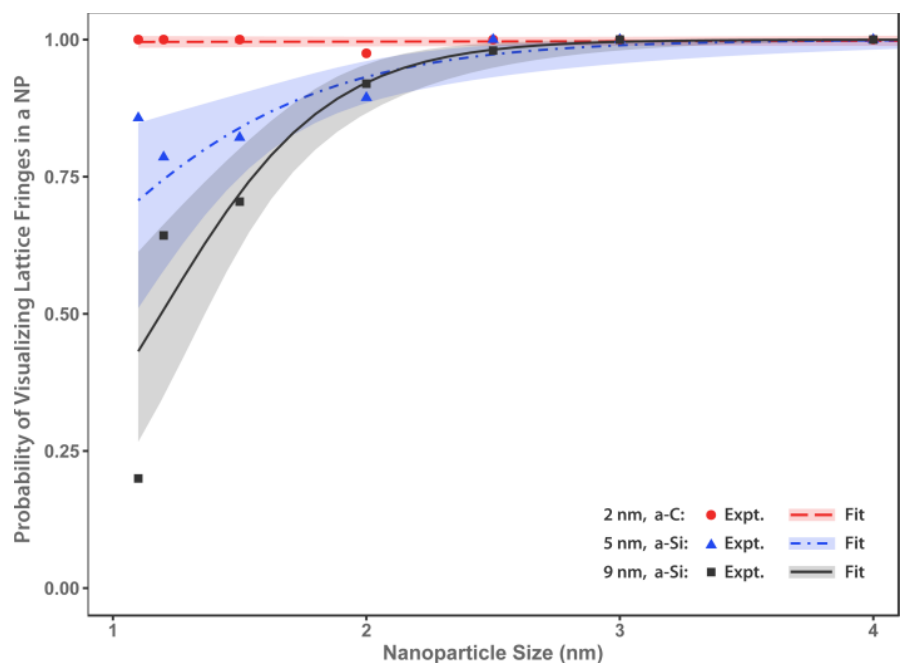

S. House et al. 


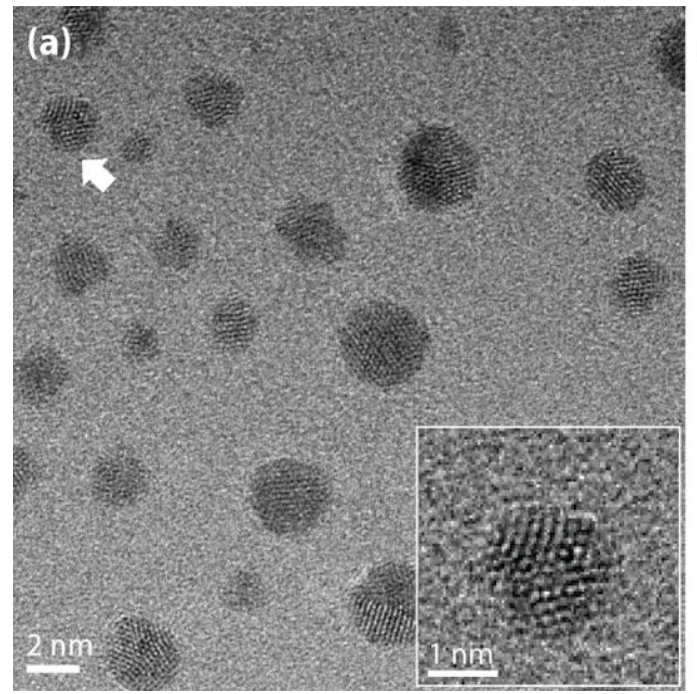

(b) $60-$ Visible $\quad \mathrm{N}=158$

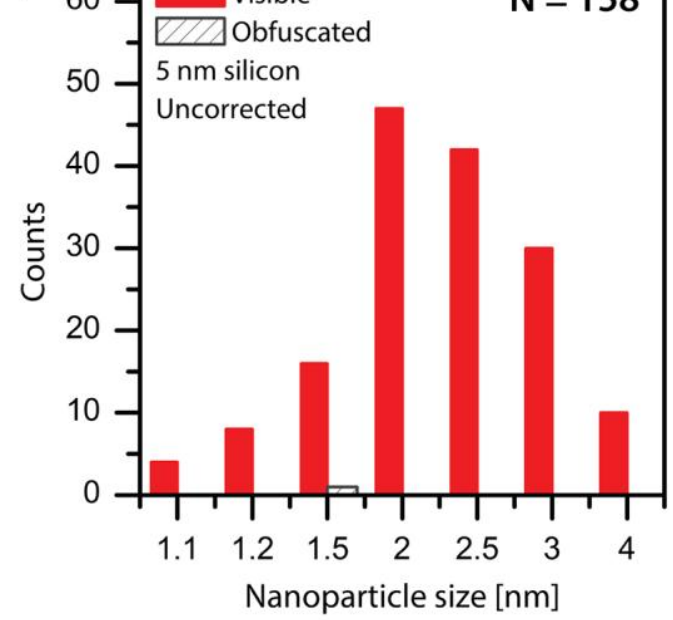

S. House et al. 


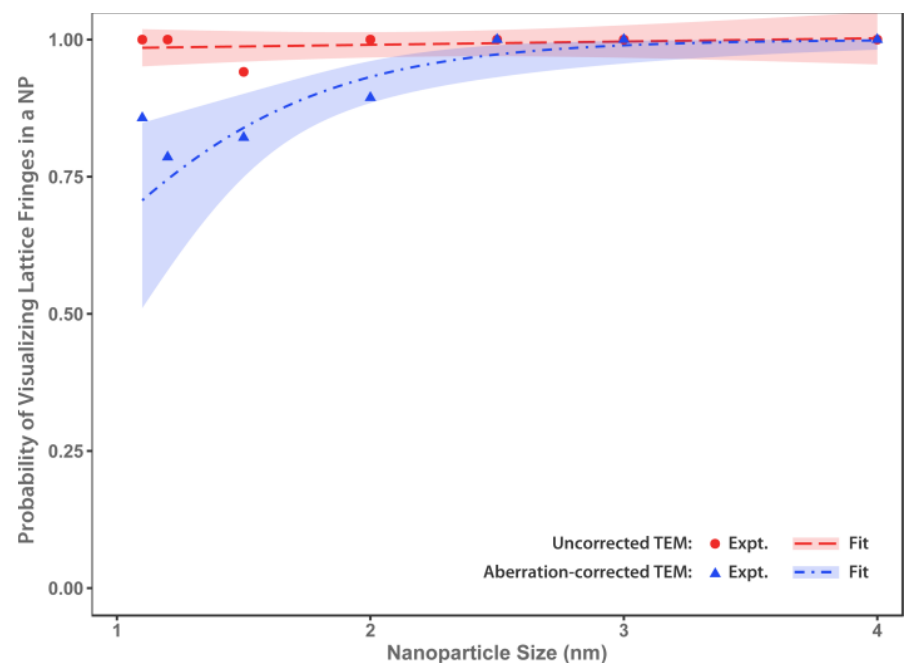

S. House et al. 

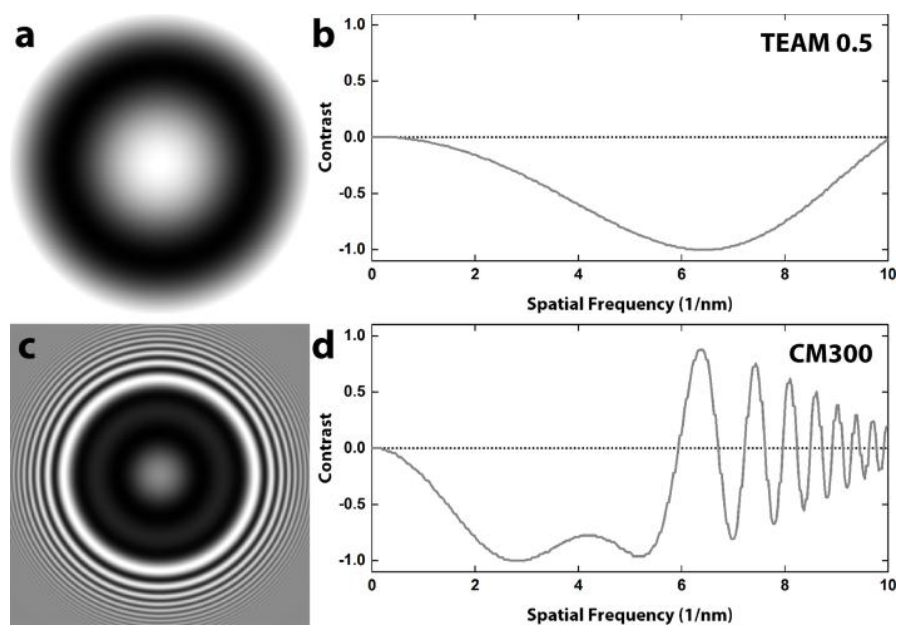

S. House et al. 